\title{
Improving undergraduate soft skills using m-learning and serious games
}

\author{
Shamus P. Smith ${ }^{1}$, Daniel Hickmott ${ }^{1}$, Ross Bille ${ }^{1}$, Elizabeth Burd ${ }^{1}$, Erica Southgate ${ }^{2}$, Liz Stephens ${ }^{2}$ \\ ${ }^{1}$ School of Electrical Engineering and Computer Science \\ ${ }^{2}$ School of Education \\ The University of Newcastle, Callaghan, NSW 2308, Australia \\ shamus.smith@newcastle.edu.au
}

\begin{abstract}
Soft skills such as effective communication are becoming increasingly important for engineering graduates. Employers prize excellent written and oral abilities and literacy proficiency. High levels of academic literacy can significantly improve students' success in their university study. Traditional approaches to literacy improvement can limit student engagement. However, mobile learning and the use of smart phone apps present new opportunities to support literacy education. This paper describes current work exploring the use of apps, as serious games, to improve literacy in undergraduate students and outlines initial results from a cross-discipline evaluation of an m-learning literacy app.
\end{abstract}

Keywords-academic literacy; literacy; communication skills; m-learning; engineering soft skills; serious games

\section{INTRODUCTION}

Effective communication is a significant contributing factor to success in many endeavors. Excellent written and oral communication skills are highly valued by employers and communication, as a soft skill, is seen as increasingly important for engineering graduates. Wang and $\mathrm{Ji}$ [1] observed that "communication and language skills are essential for engineers to carry out their professional practice".

One contributing factor to this is the widespread use of the English language in commerce. The global high-tech industry is characterized by extreme competitiveness, innovation and widespread use of English and high-tech companies require engineers that are talented in both engineering and use of English [2]. Riemer [3] observed that this will have an increasing impact on the success of engineering projects. Effective use of language to enable productive communication between professionals internationally is becoming progressively more important as the "number of international projects is increasing, and cross-cultural communication and collaboration is on the rise ... this is particularly so for the now international practice of engineering" [3].

Riemer [3] goes on to note that English is increasingly being used as the language of instruction for engineering courses in non-English speaking nations across Europe and Asia. This is also evidenced in recent literature describing international engineering programs specifically exploring how to improve English based communication skills, for example, in Thailand [4], Turkey [5] and Taiwan [2].
Engineers worldwide face numerous written English communication responsibilities involving email, reports, and memos [2]. Success in these tasks will be determined by an engineer's literacy skills. Building formal communication or academic literacy skills is a vital part of an engineering degree.

Academic literacy is defined as "the ability of students to use the English language to make and communicate meaning in spoken and written contexts while completing their university studies" [6]. Its core elements are: grammar; sentence structure; comprehension; academic writing; oral communication style; and analytical and critical thinking [7]. Research indicates that the academic literacy of undergraduate students varies considerably and that there is a clear association between academic literacy skill level and success in tertiary studies [7, $8,9]$. Poor literacy skills are associated with poor academic performance [10]. Thus, there is a need for engineering graduates to develop appropriate levels of academic literacy, both to support their success at their university studies and to equip them with appropriate communications skills for their post-university careers.

In universities, the most common approaches to developing academic literacy include the use of diagnostic tools [11], the provision of text-based (PDF, PPT) resources accessed online, and sessions with learning advisers. Resources on academic literacy are commonly text-based documents and there is currently limited use of integrated, multimodal or interactive approaches. The sector's reliance on text-based resources for delivering academic literacy information works to exclude those students whose academic literacy is poor to begin with. Referring students with poor academic literacy to sometimes dense, text-based resources they cannot read well or comprehend is not an effective way of improving their skills and may alienate them from the learning context.

An alternative approach is the use of mobile learning (mlearning) technologies and app-based serious games. Use of mobile digital devices for education is reinventing pedagogical and curriculum approaches. Mobile communication devices provide an opportunity for independent m-learning [12, 13] where students can take control of their own learning pace and engagement. Research indicates that between $60-80 \%$ of students use smart phones with around $20 \%$ also using tablet computers $[14,15]$. These numbers are increasing. Thus, the unprecedented growth in digital technologies and the educational apps they support provide a unique opportunity to 
increase engagement in learning anywhere and at any time [16].

In addition, the advent of the serious games movement has created an opportunity for educators and instructional and software designers to collaborate in the creation of learning games that incorporate the characteristics of leisure games such as fun, flexibility, competition (including self-competition) and goal mastery [17].

There are numerous m-learning apps available for teaching literacy skills at an early childhood and primary school level $[18,19,20]$. However, m-learning apps designed to improve adult literacy are less common [21].

This paper describes current work on the use of a serious game to improve academic literacy including functional literacy in undergraduate students and outlines initial results from a cross-discipline evaluation of an m-learning app. This paper is organized as follows: In Section II, we describe the app, called Apostrophe Power, which we have developed to support improved use of apostrophes. Section III provides an overview of our evaluation method and initial results from an ongoing evaluation study. Section IV provides a brief summary and draws conclusions from the current work.

\section{APOSTROPHE POWER OVERVIEW}

The purpose of the Apostrophe Power serious game app is to provide a tool that students can use to practice the correct use of apostrophes. The game includes exercises that test the use of three different functions of apostrophes, referred to as categories:

- Contractions - for example, the contraction of cannot is can't.

- Ownership - for example, singular and plural ownership, e.g. Dad's car.

- $\quad$ Misfits - the appropriate use of it's and its.

- Combination - exercises that combine the use of the previous three categories.

Lukač [22] noted that "apostrophe misuse is the most popular topic in the field of language advice when it comes to punctuation". The misuse of apostrophes in modern communications has become prevalent recently, with analysis of the language used in Twitter showing that the top five grammatical mistakes can be attributed to incorrect usage of apostrophes [22], and studies have shown that apostrophes are often misused in phone text messages [23].

The aim of the project was to develop Apostrophe Power for the two main app platforms, i.e. for iOS (Apple) and Android (Google). The goal was to provide the apps as free downloads from the app online stores for these platforms: the iTunes App Store and Google Play.

The interdisciplinary team that designed and developed Apostrophe Power consisted of researchers from the School of Education, and the School of Electrical Engineering and Computer Science (EECS) at The University of Newcastle, Australia. Studies involving the design and evaluation of educational serious games have highlighted the need for game designs to be guided by teaching theory and practice $[18,24]$. The researchers from the School of Education were experts in pedagogy and learning resource development and produced the content for the game. The researchers from the School of EECS provided the team with knowledge of software development and app technologies, insights into the gamification of content, and designed, developed, and evaluated the Apostrophe Power app.

Android and iOS versions of the Apostrophe Power app are currently available on the iTunes App Store ${ }^{1}$ and Google Play ${ }^{2}$.

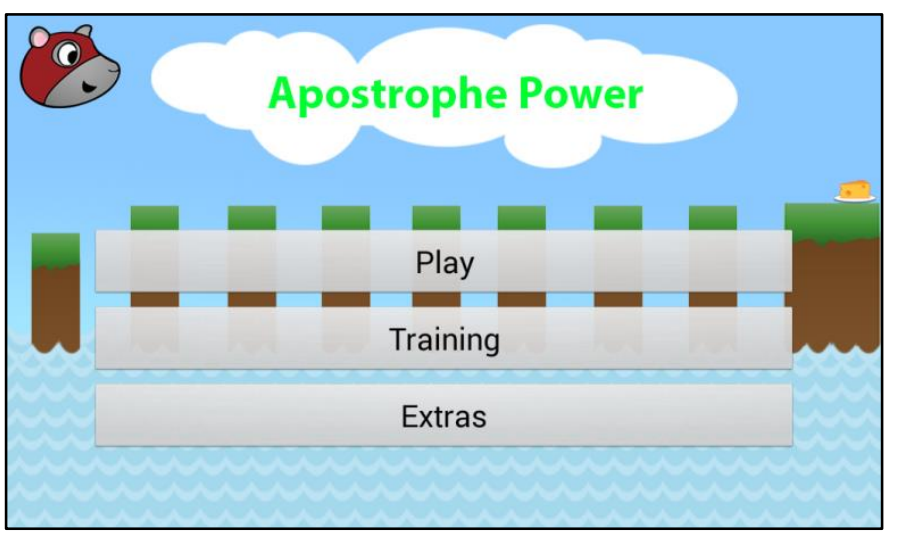

Fig. 1. The Main Menu of the Apostrophe Power game.

Fig. 1 shows the Apostrophe Power Main Menu screen that greets the user when they first start the app. The Main Menu screen is used to access to the three main sections of the game, namely Play, Training and Extras. The navigation paths from the Main Menu are shown in Fig. 2.

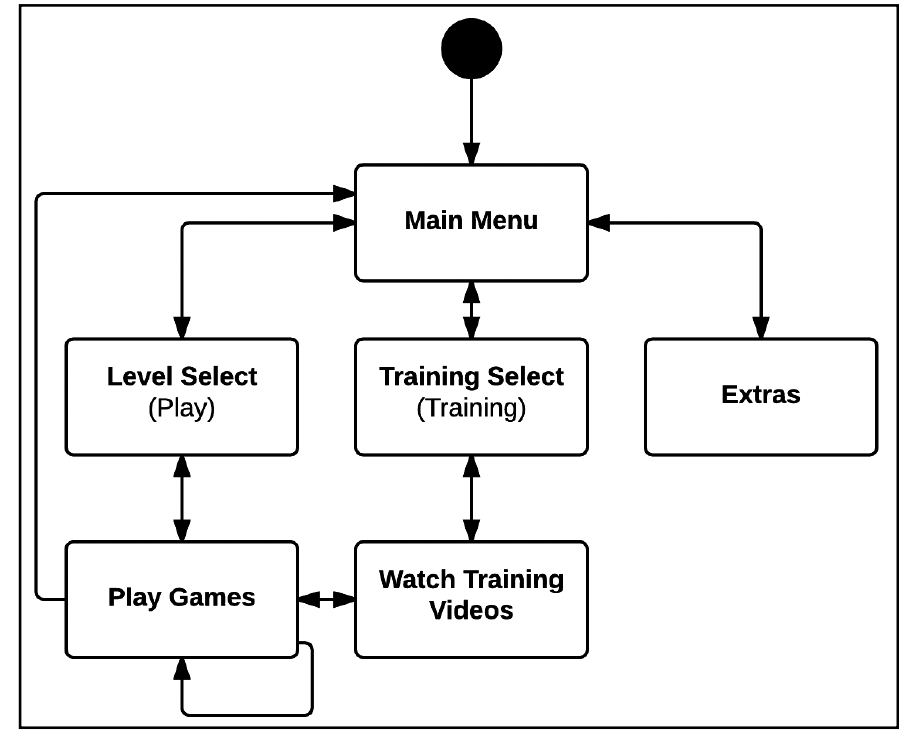

Fig. 2. The navigation paths within the Apostrophe Power game

1 https://itunes.apple.com/us/app/apostrophe-power/id1019114263 2

http://play.google.com/store/apps/details?id=com.reptiliaware.apostrophepow er 


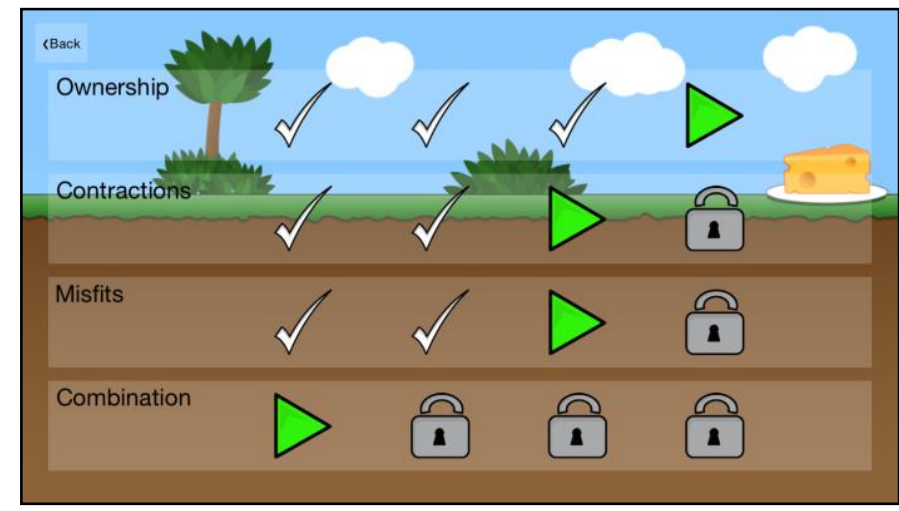

Fig. 3. A screenshot of the Level Select Menu in Apostrophe Power.

After selecting Play in the Main Menu, the player can then select a level to play using the Level Select Menu (see Fig. 3). There are four levels within each of the four categories of apostrophe usage, although only one level for each category is unlocked (available) when the game is first played. The second, third and fourth levels within each category can be unlocked by completing the preceding level. As the levels increase, so too does the difficulty of the exercises. The fourth level within each category contains a random combination of exercises from the three preceding levels. The increase in difficulty as the player completes each level is a pattern often used in education and games, referred to as scaffolding [25].

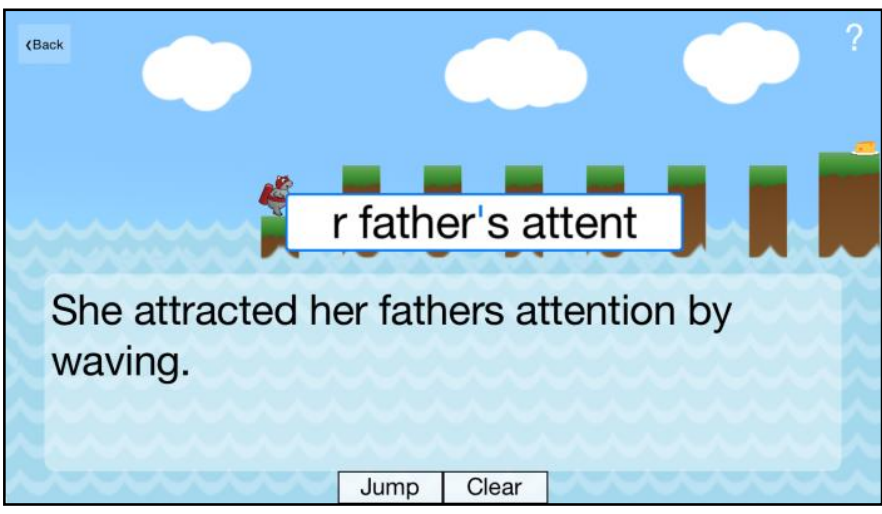

Fig. 4. A screenshot of Apostrophe Power's game play.

Selecting a level in the Level Select Menu takes a player to the main game play of the app (see Fig. 4). The goal of each level in Apostrophe Power is to move the hero - in this case, a mouse with a jetpack - from the left side of the screen to a prize, represented here by a block of cheese, on the right side of the screen. To transport the hero across the screen the player must correct the sentences presented, which are missing one or more apostrophes by tapping or dragging their finger to the location where the apostrophe/s should be. Every time the player places the apostrophe/s in the correct position/s in the sentence the hero moves to the next platform, until it reaches the prize on the last platform.

To add time pressure and motivation to each exercise, the platforms that the hero stands on slowly sink as the player attempts to correct the current sentence. If the player places the apostrophe/s in the wrong position/s in the sentence they are given the option to try another exercise. However, if they run out of time and the mouse sinks into the sea below, they are given the option to restart the level instead. In both cases, the player is also given the option to navigate back to the Main Menu or navigate to the Training section (see Fig. 5) of the app for tuition in the category of apostrophe usage that they are attempting.

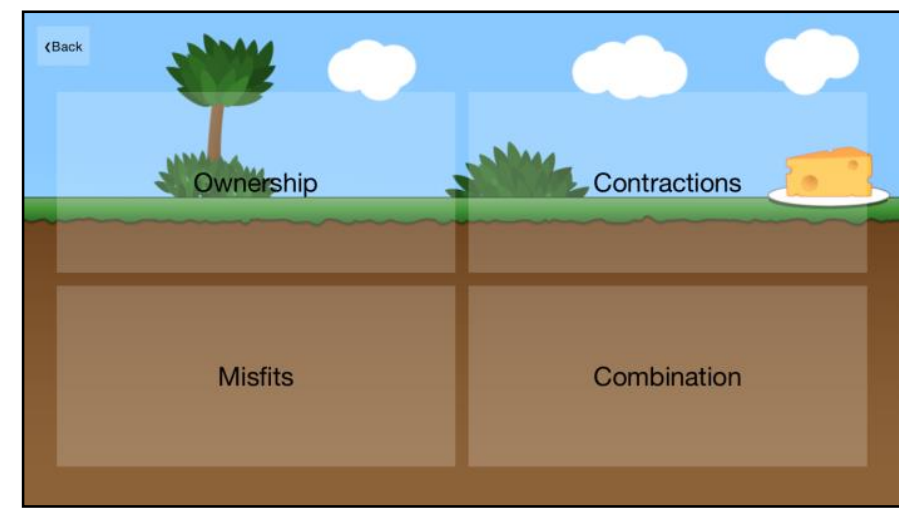

Fig. 5. A screenshot of the Training videos selection screen in Apostrophe Power.

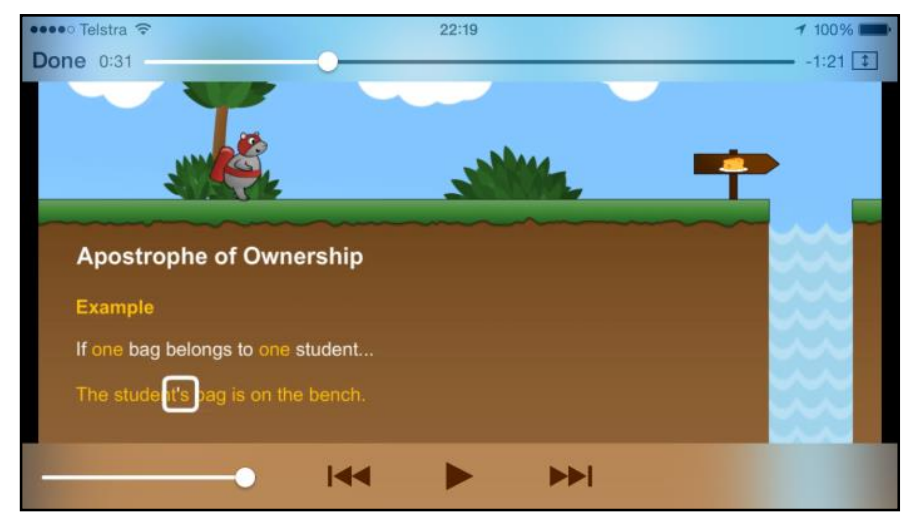

Fig. 6. A screenshot of the Ownership training video in Apostrophe Power. (Note that video controls are not typically visble and are only shown here to illustrate what is available to the player.)

The Training section consists of short video clips, approximately 1 to 2 minutes long, that provide tutorial material for each category of apostrophe featured in the game. Fig. 6 shows a screenshot of the training video for Ownership from the iOS version of Apostrophe Power. The videos were developed in Adobe Flash ${ }^{3}$ and exported as standard MP4 video files so that they would be compatible with both the Android and iOS devices and thus be reusable resources for the apps.

If the player chooses to navigate to the training videos during gameplay, after running out of time or responding to an exercise incorrectly, they are given the option to return to the level in which they made an error. This navigation path, between gameplay and training videos (shown in Fig. 2), was

3 http://www.adobe.com/au/products/flash.html (last access 05/11/2015). 
implemented in the app because it was believed that watching the videos could help players immediately discover why their responses to exercises during gameplay were incorrect.

The Extras section of the app displays the game's authors with an option for clearing the game's data, e.g. all the levels are re-locked and the recorded data (for example, the time it took for the player to complete each level) cleared for each level. A special version of the app, on the Android platform, was developed for app evaluation purposes. This version also recorded events in the game, in the form of background logging for data analytics, to show the players' navigation paths when they were completing each activity in the app. These analytics or, more specifically, game analytics, have only been included in the evaluation build of the game, and are not available in the release version to be distributed via the Google Play Store. The Extras section of the evaluation version also had an option to email out the usage logs from the app. It is this data that was collected during the evaluation of the app as described in the next section. The collection of data logs and the evaluation itself received ethics approval from The University of Newcastle (Human Research Ethics Committee approval number H-2015-0226).

\section{EVALUATION AND RESUlTS}

The evaluation of Apostrophe Power is ongoing and aims to determine the efficacy of the app for different student populations. This section describes the evaluation method and initial results.

The evaluation of the Apostrophe Power app has two parts. For part 1, undergraduate students across all disciplines at The University of Newcastle were invited, via university posters and online course advertising in a Blackboard-based web portal, to participate in an online survey to measure their current apostrophe skill level. The survey, hosted on the Lime Survey service ${ }^{4}$, was used as a screening test. The survey consisted of demographics questions, including discipline of study, and a 30-item apostrophe quiz (the pre-test) covering the three categories of apostrophe usage, i.e. ownership, contractions and misfits. There was no time limit on the survey. Students were offered entry into a prize draw at the end of the evaluation as an incentive to complete the survey. Students with scores of less than $70 \%$ in the apostrophe component of the survey were then invited to be involved in part 2 of the evaluation.

For students selected to participate in part 2, a number of game sessions were organized. During a 1 hour session, participants played the Apostrophe Power app for 40 minutes, completed a second apostrophe survey (post-test), and participated in a focus group (Note that the data from the focus groups is currently being analyzed and will not be considered here further). Students were allowed free use of the app and could attempt game activities or watch tutorial videos in any order. Students completed a post-test consisting of a 30-item apostrophe quiz with 10 questions of equivalent difficulty on each function of apostrophe use (ownership, contractions and

${ }^{4}$ http://www.limeservice.com [last access 26/10/2015]. misfits). This test was developed to be isomorphic in difficulty to the online apostrophe quiz, i.e. similar and therefore comparable to the pre-test. Students who participated in part 2 were offered a $\$ 30$ supermarket voucher to compensate them for their time.

At the time of writing, 288 students had completed part 1, the online quiz, and 131 students had scores that indicated they were eligible to be invited to part 2 of the evaluation. Initially, the intention was to use the full 30-mark score as eligibility for part 2 (e.g. only invite students with a score less than 21/30). However, on review of the results from part 1, it was found that all the students scored either 9 or 10 out of a possible 10 for the contractions questions (for example, the contraction of cannot is can 't). This significantly impacted the $70 \%$ threshold for part 2. Therefore, only the results from the ownership and misfits questions (a 14/20 threshold) have been used to determine eligibility for part 2 .

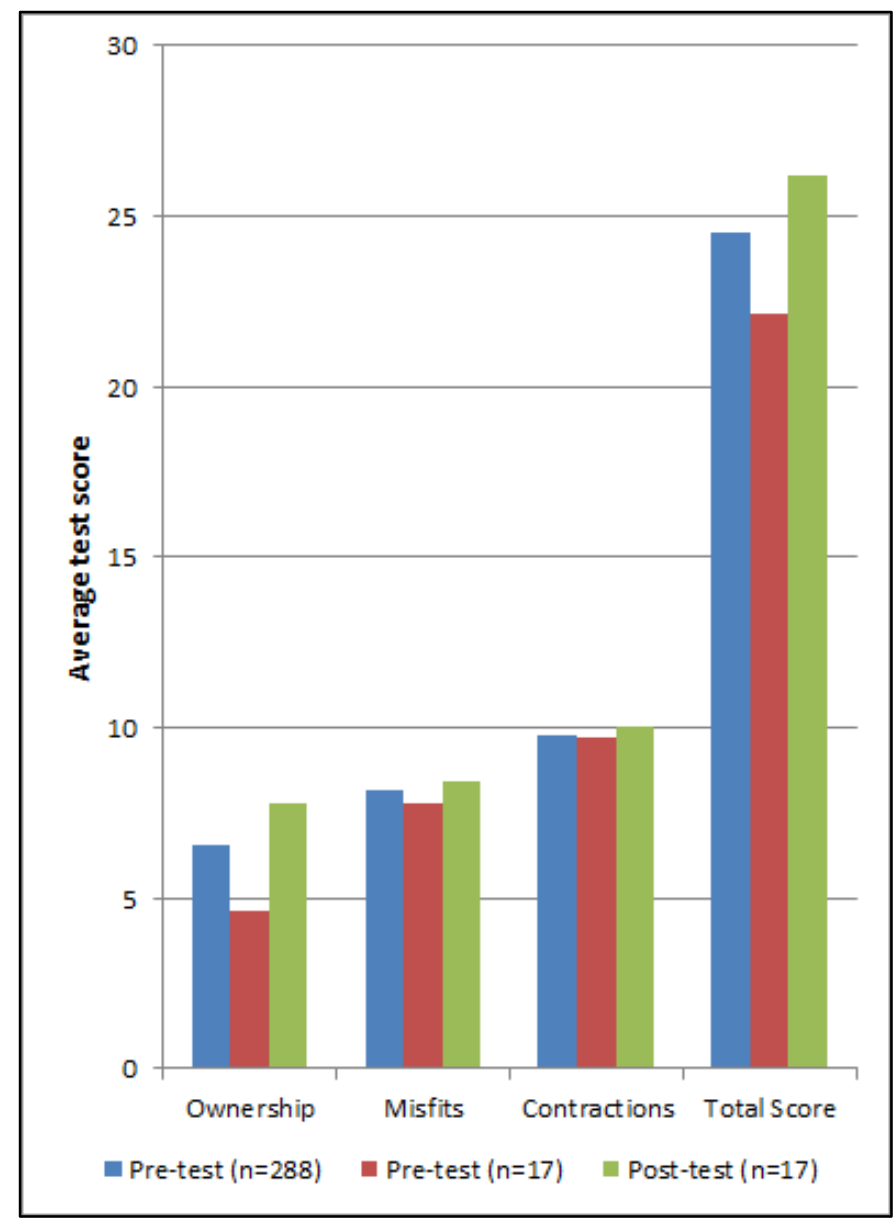

Fig. 7. Graph comparing the average pre- and post-test apostrophe quiz scores for participants from part 1 and part 2 of the Apostrophe Power evalaution.

Of the 131 students invited to be involved in part 2, seventeen have completed the app game session and post-test quiz. Participants were from a number of different disciplines, including Engineering, Education, Nursing, the Sciences and Humanities. With the current small number of participants, it is not useful to separate the participants into disciplines. 
However, an informal review of the results by discipline found similar patterns as those to be reported below. Thus the results here will consider the whole participant cohort, i.e. 288 students in part 1 and 17 students in part 2.

Although the evaluation is ongoing, initial results are promising. On average, participants who used the Apostrophe Power app improved their apostrophe usage across all test categories (as shown by the middle and right bars in each category graph in Fig. 7). In addition, prior to use of the app, participants in part 2 had, on average, lower scores across all three of the apostrophe categories, when compared to the average scores of the full pre-test cohort $(n=288)$ (shown by comparing the left bar - all the part 1 participants - with the middle bar - the part 2 participants - across categories in Fig. 7). However, after use of the app, the part 2 participants had better average scores than in the comparable post-test quiz. If the left bar of each category $(n=288)$ is considered the cohort "benchmark" for apostrophe usage of undergraduate students, the part 2 participants went from below the benchmark - the middle bar - to above the benchmark - the right bar - after one use of the app and completion of the second test. In summary, not only did the participants improve but they also progressed from below average to above average scores when compared to the larger cohort's average scores.

\section{CONCLUSION AND FUTURE WORK}

Communication as a soft skill is essential for all undergraduate students and is increasingly seen as important for engineering graduates. Correct punctuation use is a core component of literacy. There is a growing understanding that helping undergraduates to improve their literacy skills is key to mastering the technical and professional aspects of their disciplines.

The ever-increasing use of mobile technology provides exciting opportunities to engage learners. This paper describes an m-learning serious game app designed to support improvement in the use of apostrophes. A gamification approach has been used to imbed learning activities in the game-oriented app Apostrophe Power. This app is part of a suite of apps for academic literacy improvement being developed at The University of Newcastle, Australia. Initial results from an evaluation of Apostrophe Power are promising with participants from different disciplines improving their use of apostrophes.

Ongoing work includes increasing recruitment numbers of evaluation participants from different student populations to enable rigorous statistical analysis of the data collected from the evaluation. This data includes results from pre- and posttesting surveys, data analytics of gameplay and learning paths, and qualitative data from focus groups. We are also in the process of completing a second academic literacy app, called Sentence Hero, which will have activities and training videos on additional aspects of grammar and punctuation, namely full stops, commas, colons, semicolons and sentence fragments. This is ongoing work.

\section{ACKNOWLEDGMENTS}

The development of the Apostrophe Power app was supported in part by the Centre of Excellence for Equity in Higher Education, University of Newcastle, Australia (Grant \#185826036) and the Literacy App Project (HEPP National Priority Pool Grant \#188626554). Thanks also to Jeffrey Julian (UON) and Samsung for equipment support.

\section{REFERENCES}

[1] J. Y. Wang and N. N. Ji, "In case there is something fault in power supply..., then unit will broken without this," IEEE International Conf. on Teaching, Assessment and Learning for Engineering (TALE), August 2012.

[2] P. Spence and G.Z. Liu, "Engineering English and the high-tech industry: a case study of an English needs analysis of process integration engineers at a semiconductor manufacturing company in Taiwan," English for Specific Purposes, vol. 32, no. 2, pp. 97-109, April 2013.

[3] M. J. Riemer, "English and communication skills for the global engineer," Global J. Eng. Educ., vol. 6, no. 1, pp. 91-100, 2002.

[4] L. Panyawong-Ngam, N. Tangthong, and P. Anunvrapong, "A model to develop the English proficiency of Engineering students at Rajamangala University of Technology Krungthep, Bangkok, Thailand," Procedia Social and Behavioral Sciences, vol. 192, pp. 77-82, 2015.

[5] E. Gözüyeşil, “An analysis of engineering students' English language needs," Procedia - Social and Behavioral Sciences, vol. 116, pp. 41824186, 2014.

[6] DEEWR, Good practice principles for English language proficiency for international students in Australian universities - Final report. Canberra: DEEWR, 2009.

[7] N. Rolls and P. Wignell, Communicating at University: Skills for Success. 3rd ed., Darwin: CDU Press, 2009.

[8] M. E. Evans, "Planning for the transition to tertiary study: a literature review," Journal of Institutional Research, vol. 9, pp. 1-13, 2000.

[9] A. Kirkness, "Critical reflections on an academic literacies policy five years on," paper presented at the Higher Education Research and Development Society of Australia, Perth, 2006.

[10] J. Hendricks, L. Andrew, and A. C. Fowler, "The piloting of an academic literacy education course (ALEC) to improve academic literacy of first semester undergraduate students in a Western Australian University," Journal of Nursing Education and Practice, vol. 4, no. 4, pp. 19-27, 2014.

[11] H. Bonanno and J. Jones, The MASUS procedure: measuring the academic skills of university students. A diagnostic assessment. Rev. ed., University of Sydney, 2007.

[12] L. Johnson, S. Adams Becker, V. Estrada, and A. Freeman, NMC Horizon Report: 2015 Higher Education Edition. Austin, Texas: The New Media Consortium, 2015.

[13] A. Herrington, J. Herrington, and J. Mantei, "Design principles for mobile learning," in New technologies, new pedagogies: Mobile learning in higher education, J. Herrington, A. Herrington, J. Mantei, I. Olney, and B. Ferry, Eds. Wollongong: University of Wollongong, pp. 129-138, 2009.

[14] Y. Watanabe, "Ready for m-learning? Access to mobile devices by tertiary students studying Japanese," in Future challenges, sustainable futures, M. Brown, M. Hartnett, and T. Stewart, Eds., ASCILITE Conf., Wellington, pp. 1030-1038, November 2012.

[15] University of Adelaide, 2013-2017 Student e-Experience Strategy. University of Adelaide, 2013.

[16] E. Southgate, S. P. Smith, L. Stephens, D. Hickmott, and R. Billie, "Interdisciplinary opportunities and challenges in creating m-learning apps: two case studies," ASCILITE 2015, in press, November 2015.

[17] D. Charsky, "From edutainment to serious games," Games and Culture, vol. 5, no. 2, pp. 177-98, 2010.

[18] G. Falloon, "Young students using iPads: app design and content influences on their learning pathways," Computers \& Education, vol. 68, pp. 505-521, 2013. 
[19] E. Gu, T. Mo-Hong, and R. Hoda, "Fill that blank! An iOS-based literacy application," Software Engineering Conf. (ASWEC), pp. 80-83, 2014.

[20] L. Northrop and E. Killeen, "A framework for using iPads to build early literacy skills," The Reading Teacher, vol. 66, no. 7, pp. 531-537, 2013.

[21] N. Charlier, M. Ott, B. Remmele, and N. Whitton, "Not just for children: game-based learning for older adults," European Conf. on Games Based Learning, pp. 102-108, 2012.

[22] M. Lukač, “Apostrophe(')s, who needs them?” English Today, vol. 30, no. 3, pp. 3-4, 2014
[23] A. Grace, N. Kemp, F. H. Martin, and R. Parrila, "Undergraduates' attitudes to text messaging language use and intrusions of textisms into formal writing," New Media and Society, vol. 17, no. 5, pp. 792-809, 2015.

[24] K. Browne, C. Anand, and E. Gosse, "Gamification and serious game approaches for adult literacy tablet software," Entertainment Computing, vol. 5, no. 3, pp. 135-146, 2014.

[25] G. Gunter, R. Kenny, and E. Vick, "A case for a formal design paradigm for serious games," Journal of the International Digital Media and Arts Association, vol. 3, no. 1, pp. 1-19, 2006. 